\title{
DIRECT DETECTION AND ORBITAL ANALYSIS OF THE EXOPLANETS HR 8799 bcd FROM ARCHIVAL 2005 KECK/NIRC2 DATA
}

\author{
Thayne Currie $^{1}$, Misato Fukagawa $^{2}$, Christian Thalmann $^{3}$, Soko Matsumura $^{4}$, and Peter Plavchan ${ }^{5}$ \\ ${ }^{1}$ NASA-Goddard Space Flight Center, Greenbelt, MD, USA \\ ${ }^{2}$ Department of Earth and Space Science, Graduate School of Science, Osaka University, Osaka, Japan \\ ${ }^{3}$ Astronomical Institute Anton Pannekoek, University of Amsterdam, 1090-GE, The Netherlands \\ ${ }^{4}$ Department of Astronomy, University of Maryland-College Park, MD 20742, USA \\ ${ }^{5}$ NASA Exoplanet Science Institute, California Institute of Technology, Pasadena, CA, USA \\ Received 2012 May 30; accepted 2012 July 5; published 2012 August 7
}

\begin{abstract}
We present previously unpublished 2005 July $H$-band coronagraphic data of the young, planet-hosting star HR 8799 from the newly released Keck/NIRC2 archive. Despite poor observing conditions, we detect three of the planets (HR $8799 \mathrm{bcd}$ ), two of them (HR $8799 \mathrm{bc}$ ) without advanced image processing. Comparing these data with previously published 1998-2011 astrometry and that from re-reduced 2010 October Keck data constrains the orbits of the planets. Analyzing the planets' astrometry separately, HR 8799 d's orbit is likely inclined at least $25^{\circ}$ from face-on and the others may be on inclined orbits. For semimajor axis ratios consistent with a 4:2:1 mean-motion resonance, our analysis yields precise values for HR 8799 bcd's orbital parameters and strictly constrains the planets' eccentricities to be less than $0.18-0.3$. However, we find no acceptable orbital solutions with this resonance that place the planets in face-on orbits; HR $8799 \mathrm{~d}$ shows the largest deviation from such orbits. Moreover, few orbits make HR $8799 \mathrm{~d}$ coplanar with b and c, whereas dynamical stability analyses used to constrain the planets' masses typically assume coplanar and/or face-on orbits. This Letter illustrates the significant science gain enabled with the release of the NIRC2 archive.
\end{abstract}

Key words: planetary systems - stars: early-type - stars: individual (HR 8799)

Online-only material: color figures

\section{INTRODUCTION}

The nearby, young A-type star HR $8799(d=39.4$ pc, $\approx 30 \mathrm{Myr}$; Zuckerman et al. 2011) harbors the first independently confirmed, directly imaged exoplanetary system and the only imaged multi-planet system (Marois et al. 2008). After the discovery of HR 8799 bcd $\left(r_{\mathrm{proj}} \approx 24,38\right.$, and $68 \mathrm{AU}$ ) reported in 2008 November (Marois et al. 2008), other studies identified at least one of these planets in archival data taken prior to 2008 (Lafreniére et al. 2009; Fukagawa et al. 2009; Metchev et al. 2009; Soummer et al. 2011).

HR 8799 planet astrometry derived from both pre- and postdiscovery images can help constrain the system's dynamical stability and, in turn, the planets' physical properties. At least two of the HR 8799 planets are likely locked in a meanmotion resonance, otherwise the system would quickly become dynamically unstable (Fabrycky \& Murray-Clay 2010). The recently discovered fourth companion at $\sim 15 \mathrm{AU}, \mathrm{HR} 8799 \mathrm{e}$, generally makes dynamical stability less likely (Marois et al. 2010b; Currie et al. 2011a), favoring lower masses of $M_{\mathrm{b}, \text { cde }}<$ $7,10 M_{\mathrm{J}}$, an important constraint given the uncertainties in deriving masses from planet cooling and atmosphere models (Spiegel \& Burrows 2012; Madhusudhan et al. 2011).

Studies focused on fitting the planets' orbits and/or testing dynamical stability typically assume that the planets are (1) in resonance (4:2:1 for HR 8799 bcd or 2:1 for HR $8799 \mathrm{~cd}$ ); (2) in circular, face-on orbits; (3) and/or in coplanar orbits (e.g., Marois et al. 2010b; Currie et al. 2011a; see also Fabrycky and Murray-Clay 2010). However, Soummer et al. (2011) show that circular, face-on, and coplanar orbits are inconsistent with 1998 Hubble Space Telescope (HST) astrometry, identifying a best-fit orbit for HR $8799 \mathrm{~d}$ of $i=28^{\circ}$ and $e=0.115$. Generally, more eccentric orbits destabilize the system. The system stability depends on the (mutual) inclinations of the planets (Fabrycky \& Murray-Clay 2010; Sudol \& Haghighipour 2012). Thus, the HR 8799 planets' true mass limits derived from dynamical stability arguments may slightly differ from those previously reported.

Well-sampled HR 8799 d astrometry could help clarify whether HR 8799 d's orbit must be inclined, eccentric, and/or coplanar with the other planets. However, until now there is a $\sim 9$ year gap between the 1998 HST detection and the next one (2007; Metchev et al. 2009). New astrometry for HR 8799 bce in between 1998 and 2007 could also help constrain those planets' orbits. By better determining the HR 8799 planets' orbital properties, we can more conclusively investigate system dynamical stability and thus better clarify the range of allowable planet masses.

In this Letter, we report the detection of HR 8799 bcd from unpublished, now-public Keck/NIRC2 data taken in 2005 supplemented with a re-reduction of published 2010 October data from Marois et al. (2010b). We use these data to better constrain the orbital properties of HR 8799 bcd.

\section{OBSERVATIONS AND DATA REDUCTION}

\subsection{July Data}

We downloaded HR 8799 data taken on 2005 July 15 from the newly available Keck/NIRC2 data archive (Program ID H53BN2, PI: Michael Liu). The data were taken in the $H$ band with the narrow camera (9.952 mas pixel ${ }^{-1}$; Yelda et al. 2010) with the 0 "' 6 diameter coronagraphic spot and the "incircle" pupil plane mask. HR 8799 was observed in $10 \mathrm{~s}$ exposures in "vertical angle" or angular differential imaging mode (Marois 

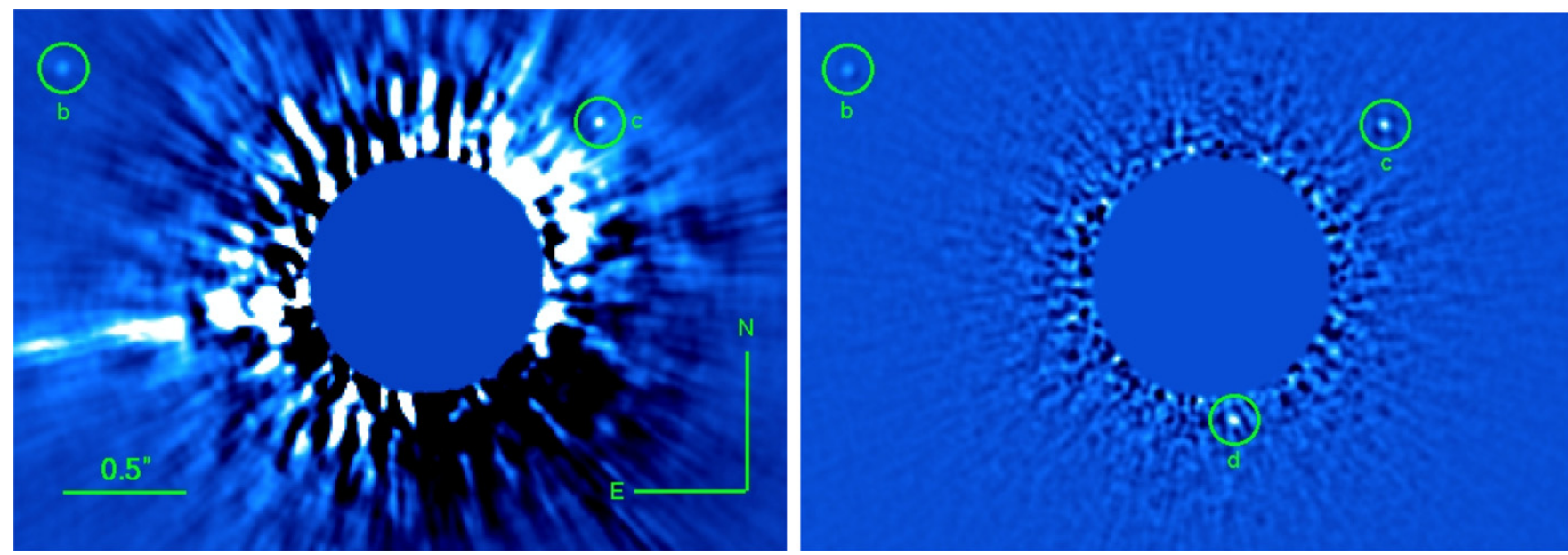

Figure 1. HR 8799 images processed with "classical" PSF subtraction (left) and A-LOCI (right) showing the detections of HR 8799 b, c, and d (circled). (A color version of this figure is available in the online journal.)

et al. 2006) through transit (hour angle $=[-0.20,0.55]$ ) with a total field rotation of 147.1 . During these observations, the seeing conditions fluctuated and the observer's periodically recentered the star behind the mask, changing the intensity profile of the stellar halo (and thus the quasi-static speckle pattern). During a few frames near transit the star did not properly center at all behind the coronagraph. We identify $\sim 11$ minutes of science-grade data. Basic image processing followed standard steps previously used to process NIRC2 data (Currie et al. 2012).

For a first-order reduction, we perform a simple "classical" ADI-based point-spread function (PSF) subtraction (e.g., Marois et al. 2006). Figure 1 shows this reduction, clearly revealing HR $8799 \mathrm{~b}(\mathrm{~S} / \mathrm{N} \sim 12)$ and identifying HR $8799 \mathrm{c}$, albeit at low $\mathrm{S} / \mathrm{N}(\sim 4)$. With the LOCI approach (Lafrenière et al. 2007) as implemented and modified in previous work (Currie et al. 2010, 2011a, 2011b), we easily detect HR 8799 b and c and obtain a marginal detection of HR $8799 \mathrm{~d}$ at $r \sim 0$.' 6 several degrees away (clockwise) from the 2007 July position reported by Metchev et al. (2009; not shown).

To improve the signal to noise ratio $(\mathrm{S} / \mathrm{N})$ of our HR $8799 \mathrm{~d}$ detection, we incorporated several upgrades to enhance contrast which are being implemented in a new "adaptive" LOCI (A-LOCI) pipeline (T. Currie, 2012 in preparation; see also similar steps in Marois et al. 2010a). We subtract off the seeing halo in each image to measure the static/quasi-static speckle pattern, determining the cross-correlation function for the speckle patterns in annular sections for all possible image pairs. While the speckle pattern is generally better correlated between frames taken close together in time, this is not always the case, especially when comparing frames before and after small telescope nods. Therefore, for each annular section of a science image we want to subtract, we filter reference image sections by their degree of correlation to remove those below a certain, predetermined threshold $\left(r_{\text {corr }}\right)$.

HR $8799 \mathrm{~b}$ is detectable in most individual processed frames (S/N/frame 4-7), so we measure its position to identify and correct for any astrometric biases caused by a position angle (P.A.) "jump" for frames obtained near transit due to imperfect mechanical alignment of the telescope's $y$ axis. The P.A.s of HR $8799 \mathrm{~b}$ in frames more than $0.25 \mathrm{hr}$ from transit are consistent, but the P.A. offset follows a bell-shaped curve with a maximum offset of $\approx 0.6$ centered on transit. We model and correct for this offset using a fifth-order polynomial. We also reran our pipeline with different rotation axis offsets due to image registration errors, setting an upper limit to this of 0.5 pixels in each coordinate.

Furthermore, unlike the original LOCI algorithm, we set the azimuthal length of the subtraction zone to be smaller (not identical) to the azimuthal length for the optimization zone, equal to $d r$ (the length along the radial direction). We then center the optimization zone on the subtraction zone. Finally, we iteratively determine the algorithm parameters- $\delta, N_{A}, g$, $d r$ (Lafrenière et al. 2007), and $r_{\text {corr }}$ that maximize the $\mathrm{S} / \mathrm{N}$ of point sources and applied these settings to extract our final image. 6

Figure 1 (right panel) shows our final image displaying higher $\mathrm{S} / \mathrm{N}$ detections of $\mathrm{HR} 8799 \mathrm{~b}(\mathrm{~S} / \mathrm{N} \sim 38)$ and $\mathrm{c}(\mathrm{S} / \mathrm{N} \sim 18)$ and new detection of $\mathrm{d}(\mathrm{S} / \mathrm{N} \sim 5)$, using algorithm parameters of $\delta \geqslant 0.74, N_{A}=245, g=0.95, d r=6$, and $r_{\text {corr }} \geqslant 0.315$, though similar settings yield nearly identical results (i.e., $\delta \geqslant$ $\left.0.73-0.8, r_{\text {corr }} \geqslant 0.31-0.36\right)$. We achieve contrast gains of up to $\sim 80 \%$ (for HR 8799 d) over our best LOCI reduction.

For flux calibration, we perform aperture photometry on HR 8799 bcd and on the nearby star GJ 616.2 observed just prior to HR 8799. We use fake point sources to further correct for LOCI-based photometric biases (i.e., Lafrenière et al. 2007; Currie et al. 2011a, 2011b) and find $m(H)=18.05 \pm 0.09 \mathrm{mag}$ for HR $8799 \mathrm{~b}, m(H)=17.06 \pm 0.13$ for HR $8799 \mathrm{c}$, and $m(H)=16.71 \pm 0.24$ for HR $8799 \mathrm{~d}$. The magnitude differences between HR $8799 \mathrm{c}$ and d appear slightly discrepant compared to better calibrated NIRC2 measurements from Marois et al. (2008), although the individual measurements are consistent to within $\sim 1 \sigma$. Photometry derived for HR 8799 bc using classical PSF subtraction agrees with that derived from our A-LOCI based reduction within errors. Bright residual speckles at $r=0$ '.3-0.'4 prevent detecting HR 8799 e.

\subsection{October Data}

To supplement the 2005 HR 8799 astrometry, we downloaded and reduced 2010 October $L^{\prime}$-band NIRC2 data from the Keck archive (PI: B. Macintosh). These data are the latest reported by

\footnotetext{
6 We also considered a "reference PSF library" derived from other 2004-2005 NIRC2 data obtained with the same setup (coronagraph size, filter, etc.) to further attenuate speckles. However, this library degrades the $\mathrm{S} / \mathrm{N}$ of HR 8799 b and $\mathrm{c}$ by $\approx 30 \%-60 \%$ and renders HR 8799 d undetectable, because HR 8799's speckle patterns are poorly correlated with library's.
} 
Marois et al. (2010b) who focus on HR 8799 e astrometry: We use these data instead to extract astrometry for HR $8799 \mathrm{bcd}$. Individual exposures consist of $50 \mathrm{~s}$ frames totaling $\approx 80$ minutes taken through transit without a coronagraph with periodic telescope nods for sky subtraction. Observing conditions appeared variable at a level comparable to the 2005 July data and worse than other recent Keck data (i.e., Currie et al. 2012; Rodigas et al. 2012), but we detect all four planets $(\mathrm{S} / \mathrm{N} \sim 6-20)$ with our pipeline.

\section{ASTROMETRIC ANALYSIS AND ORBIT FITTING}

\subsection{Method}

We use our detections to better constrain the orbits of HR $8799 \mathrm{bcd}$, first by fitting the orbits of the planets separately, then identifying the subset of orbits consistent with systematically more stable mean-motion resonance configuration. We calibrate our astrometry by assessing and correcting for biases introduced by LOCI-based processing in the same manner as our photometric calibration, using fake point sources. Using different telescopes and slightly different image processing techniques lead to systematic biases in planet astrometry. To minimize these biases, we restrict ourselves to astrometry from HST/1998 (Soummer et al. 2011), Subaru/2002 and 2009 (Fukagawa et al. 2009; Currie et al. 2011a), Keck/2004, 2005, 2007-2009 (Marois et al. 2008; Metchev et al. 2009; Galicher et al. 2011, this work), VLT/2009 (Currie et al. 2011a), and LBT/Pisces (Esposito et al. 2012). For the Pisces data, we include the substantial north P.A. uncertainty. We furthermore modify the Keck/NIRC2 astrometry reported in Marois et al. (2008) and Metchev et al. (2009) to reflect the updated NIRC2 astrometric calibration (Yelda et al. 2010) accuracy, rescaling the position by a factor of $9.952 / 9.963$, and putting in a P.A.north,new - P.A.north,old $=0.13$ clockwise rotation.

To separately determine the range of allowable HR 8799 bcd orbits, we follow our previously adopted Monte Carlo based approach (Thalmann et al. 2009; Currie et al. 2011b), comparing the HR 8799 planet astrometry to predictions from randomly selected orbits. In a first set of "conservative" simulations, we consider the orbits separately. Dynamical stability analysis suggests that HR 8799 bcd are likely in resonance (Fabrycky \& Murray-Clay 2010); the 4:2:1 resonance is particularly adept at stabilizing the system. To focus on dynamically stable orbits we then select the subset of best-fitting orbits that preclude the planets from crossing orbits and are consistent with a 4:2:1 resonance. Here we define "resonance" broadly, including orbits with ratios of periods between 1.9 and 2.1 for consecutive pairs of planets since, at least in some circumstances, exact period ratios may be rare (e.g., Fabrycky et al. 2012). ${ }^{7}$

For all our simulations, the minimum $\chi^{2}$ for the $b, c$, and $d$ planets are 20.8, 13.8, and 14.8, for reduced $\chi_{v}^{2}$ values of 1.04, 0.86, and 1.24. Following Currie et al. (2011b), we choose a cutoff of $\chi^{2} \leqslant \chi_{\min }^{2}+1$ to represent the family of best-fitting orbits. Formally, this cutoff admits only an average additional deviation per $x$ or $y$ measurement of $\approx 1 / 2 N_{\text {obs }}$ (i.e., $\approx 1 / 20$ for HR $8799 \mathrm{~b}$; $\approx 1 / 14$ for HR 8799 d) beyond the best-fit models, which themselves imply typical deviations of $\approx 1 \sigma$ per each $x$ or $y$ measurement (since the minimum reduced $\chi_{v}^{2}$ values are $\approx 1$ ). However, we obtain nearly identical results for more relaxed cutoffs (see below). From the set of models passing

\footnotetext{
7 Dynamical simulations identify stable solutions for at least some planet masses where only HR $8799 \mathrm{c}$ and d are in resonance, so our conclusions from this set of astrometric analyses may be less applicable for HR 8799 b.
}

our $\chi^{2}$ cutoff, including the subset in resonance, we determine the weighted median value and the weighted $68 \%$ confidence interval about the median for each model parameter from among the set of best-fitting orbits.

Furthermore, we report a "most likely orbit" (MLO) simply defined as follows. First, over the best-fitting family of orbits, we calculate histograms for the following parameters: logarithm of the semimajor axis $\left(h_{\log a}\right)$, eccentricity $\left(h_{e}\right)$, inclination $\left(h_{i}\right)$, longitude of ascending node $\left(h_{\Omega}\right)$, and argument of periastron $\left(h_{\omega}\right)$. For each orbit $n$ in the best-fitting ensemble, we then define the measure of likelihood $\mathcal{L}(n)$ as

$$
\mathcal{L}(n)=h_{\log a}\left(\log a_{n}\right) \cdot h_{e}\left(e_{n}\right) \cdot h_{i}\left(i_{n}\right) \cdot h_{\Omega}\left(\Omega_{n}\right) \cdot h_{\omega}\left(\omega_{n}\right) \cdot W,
$$

i.e., the product of all histogram values in the bins in which the orbit $n$ lies, representing the individual likelihood of each measured orbital parameter, as well as the statistical weight, $W$, representing the likelihood of the observed planet position within the orbit (i.e., the anomaly). ${ }^{8}$ The MLO is then the one orbit that maximizes the measure of likelihood, $\mathcal{L}\left(n_{\mathrm{MLO}}\right)=$ $\max _{n} \mathcal{L}(n)$. Because of the highly skewed distribution of some parameters from the best-fit orbits, in particular $\log a$, the MLO parameters can differ significantly from the weighted median parameters.

\subsection{Results}

Table 1 summarizes our results, and Figure 2 displays the orbits in $a / i / e$ space (left), the histogram distribution of $i$ (middle), and the histogram distribution of the longitude of the ascending node, $\Omega$ (right). The top panels display properties for HR 8799 b, the middle for HR $8799 \mathrm{c}$, and the bottom for HR 8799 d. Assuming a $\chi^{2}$ cutoff of $\chi_{\min }^{2}+1$, the observations constrain the HR 8799 d orbit well $-a \sim 24-32$ AU, $i \sim$ $32^{\circ}-42^{\circ}, e \sim 0.03-0.23$, and $\Omega \sim 43^{\circ}-63^{\circ}$. They limit the HR 8799 c's most plausible orbital parameters to $a \sim 36-42 \mathrm{AU}$, $i \sim 13^{\circ}-26^{\circ}$, and $e \sim 0.03-0.13$. As expected, the parameters for HR $8799 \mathrm{~b}$ are the most poorly constrained, showing the widest dispersion and the largest differences between the median parameter value and that from the MLO.

Our analysis clearly disfavors face-on orbits for all three planets, especially for HR $8799 \mathrm{c}$ and d. Additionally, the inclination distributions for HR 8799 bc appear systematically skewed toward values lower than those for d. Formally, though, the set of acceptably fitting orbits HR 8799 bcd includes some that make the planets coplanar.

Assuming a 4:2:1 mean-motion resonance configuration, we place far stronger limits on nearly all of the HR 8799 bcd orbital properties (Figure 3). This assumption explicitly rules out $e>$ 0.18 for HR 8799 bc and $e>0.3$ for HR 8799 d. Likewise, we identify a very narrow range of planet semimajor axes: $a=$ 67.5-70.8 AU, 42.1-44.4 AU, and 26.4-28.1 AU for HR 8799 b, $c$, and d. The $68 \%$ confidence interval in $\Omega$ for HR $8799 \mathrm{~d}$ further narrows to $46^{\circ}-62^{\circ}$.

For this configuration, HR 8799 d (c) must be in an orbit viewed more than $25^{\circ}\left(15^{\circ}\right)$ from face-on while HR $8799 \mathrm{~b}$ is likely inclined by at least $5^{\circ}$. Furthermore, the inclination distributions between HR 8799 d and HR 8799 bc are even more dissimilar, implying that HR $8799 \mathrm{~d}$ is most likely inclined relative to $\mathrm{c}$ by at least $\approx 7^{\circ}$ and b by more than $\approx 21^{\circ}$. While our analyses cannot conclusively rule out coplanar orbits in a

\footnotetext{
8 Here, the statistical weight $W$ is defined as the mean orbital velocity for the corresponding orbit divided by the orbital velocity at the observed epoch, $W:=\langle v\rangle_{\text {orbit }} / v\left(t_{\text {obs }}\right)$.
} 
Table 1

HR 8799 Planet Photometric/Astrometric Properties

\begin{tabular}{|c|c|c|c|}
\hline Parameter & HR 8799 b & HR 8799 c & HR 8799 d \\
\hline \multicolumn{4}{|l|}{ Measured } \\
\hline \multicolumn{4}{|l|}{ Photometry } \\
\hline$m(H)$ on $2005 \mathrm{Jul} 15$ & $18.05 \pm 0.09$ & $17.06 \pm 0.13$ & $16.71 \pm 0.24$ \\
\hline \multicolumn{4}{|l|}{ Astrometry } \\
\hline $2005 \mathrm{Jul} 15([\mathrm{E}, \mathrm{N}])^{\prime \prime}$ & $1.496,0.856( \pm 0.005)$ & $-0.713,0.630( \pm 0.005)$ & $-0.087,-0.578( \pm 0.010)$ \\
\hline 2010 Oct $30([\mathrm{E}, \mathrm{N}])^{\prime \prime}$ & $1.546,0.748( \pm 0.005)$ & $-0.598,0.737( \pm 0.005)$ & $-0.283,-0.567( \pm 0.005)$ \\
\hline \multicolumn{4}{|l|}{ Derived } \\
\hline \multicolumn{4}{|l|}{ MLO, med., [68\% C.I.] } \\
\hline \multicolumn{4}{|l|}{$\left(\chi_{\lim }^{2} \leqslant \chi_{\min }^{2}+1\right)$} \\
\hline$a(\mathrm{AU})$, full & $71.0,109.9[69.7,164.9]$ & $37.2,38.0[35.5,42.0]$ & $26.2,27.3[24.4,31.5]$ \\
\hline // 4:2:1 resonance & $68.1,68.8[67.5,70.8]$ & $42.5,43.2[42.1,44.4]$ & $27.3,27.3[26.4,28.1]$ \\
\hline$i\left(^{\circ}\right)$, full & $14.1,34.9[12.3,43.0]$ & $21.7,19.9[12.5,25.8]$ & $37.1,37.9[31.6,41.6]$ \\
\hline // 4:2:1 resonance & $8.5,9.5[4.9,14.8]$ & $25.8,27.6[25.2,28.8]$ & $38.1,37.9[36.0,39.1]$ \\
\hline$e$, full & $0.02,0.27[0.02,0.49]$ & $0.01,0.08[0.03,0.13]$ & $0.04,0.09[0.03,0.23]$ \\
\hline /I 4:2:1 resonance & $0.01,0.02[0.0,0.03]$ & $0.12,0.14[0.11,0.17]$ & $0.03,0.04[0.01,0.08]]$ \\
\hline$\Omega\left({ }^{\circ}\right)$, full & $149.7,141.0[40.9,161.4]$ & $128.8,122.2[62.0,152.4]$ & $56.8,53.5[43.3,63.0]$ \\
\hline /I 4:2:1 resonance & $163.2,87.1[22.9,158.9]$ & $147.4,131.9,[104.8,158.5]$ & $56.9,54.3[46.0,60.2]]$ \\
\hline \multicolumn{4}{|l|}{$\left(\chi_{\lim }^{2} \leqslant \chi_{\min }^{2}+[5,5,3.5]\right)$} \\
\hline$a(\mathrm{AU})$, full & $70.7,80.8[68.2,117.2]$ & $38.2,39.8[36.6,46.2]$ & $26.2,27.0[23.0,31.0]$ \\
\hline // 4:2:1 resonance & $68.0,68.5[66.4,71.0]$ & $42.7,42.9[41.3,44.6]$ & $27.3,27.1[26.0,28.2]$ \\
\hline$i\left(^{\circ}\right)$, full & $15.2,24.5[10.4,36.6]$ & $19.7,23.2[14.0,31.3]$ & $38.1,36.9[27.1,41.2]$ \\
\hline // 4:2:1 resonance & $10.8,11.5[6.1,17.1]$ & $28.8,27.4[24.6,30.2]$ & $37.1,37.7[34.6,39.9]$ \\
\hline$e$, full & $0.01,0.13[0.02,0.34]$ & $0.01,0.07[0.02,0.15]$ & $0.01,0.15[0.04,0.32]$ \\
\hline /I 4:2:1 resonance & $0.01,0.03[0.01,0.06]$ & $0.02,0.09[0.02,0.14]$ & $0.0,0.05[0.01,0.11]$ \\
\hline$\Omega\left({ }^{\circ}\right)$, full & $142.7,119.5[32.4,160.5]$ & $124.7,86.8[37.1,142.5]$ & $52.5,58.4[41.0,98.5]$ \\
\hline /I 4:2:1 resonance & $142.1,78.2[21.9,154.3]$ & $61.1,70.5[42.2,140.5]$ & $60.9,55.7[44.1,67.2]$ \\
\hline
\end{tabular}

Notes. Measured parameters: our photometric uncertainties consider both the signal to noise of our detections and the absolute flux calibration uncertainties; astrometric uncertainties consider the $\mathrm{S} / \mathrm{N}$, astrometric calibration uncertainty (e.g., 0.5 pixels in $x$ and $y$, see Section 2), etc. Derived parameters: the three column entries are MLO (the "most likely orbit" (see Section 3), med. (the median parameter value), and [68\% C.I.] (the $68 \%$ confidence interval).

4:2:1 mean-motion resonance, they suggest that few such orbits are compatible with 12 years of HR 8799 planet astrometry.

To confirm that we are fully sampling the subset of orbital parameters covering the $\chi^{2}$ minima, we run our simulations with a more relaxed $\chi^{2}$ cutoff of $\chi^{2} \leqslant \chi_{\min }^{2}+[5,5,3.5]$ for HR $8799 \mathrm{~b}, \mathrm{c}$, and d, which formally admit an additional average deviation from the data of $\approx 0.3 \sigma$ for each measurement. With this cutoff, we obtain nearly identical results (second set of rows in Table 1). Considering the planets' orbits separately, we find $a \sim 23-31 \mathrm{AU}, i \sim 27^{\circ}-41^{\circ}, e \sim 0.01-0.31$, and $\Omega \sim 41^{\circ}-53^{\circ}$ for HR $8799 \mathrm{~d}$. We find similar ranges in orbital parameters for HR 8799 c and (for the resonance case) HR 8799 b. Likewise, the planets' range of inclinations exclude faceon orbits. HR 8799 d's inclination distribution is skewed to systematically higher values, expected if it is non-coplanar with $\mathrm{b}$ and $\mathrm{c}$, although here there are more orbit combinations that could make the planets coplanar.

\section{DISCUSSION}

From analyzing HR 8799 bcd astrometry from our new "pre-discovery" image and other data, we provide new constraints on the planets' orbital properties. Treating the three planets separately, we narrowly constrain three major orbital parameters $(a / i / e)$ for HR $8799 \mathrm{c}$ and d. None of the planets are likely to be orbiting face-on and the inclinations for acceptably fitting orbits are systematically higher for HR $8799 \mathrm{~d}$ than for HR $8799 \mathrm{~b}$ and $\mathrm{c}$.
If HR 8799 bcd have semimajor axes consistent with a 4:2:1 resonance, our analysis strongly constrains the major orbital properties for all three planets. The three planets (especially c and d) then even more obviously have inclined orbits. Most acceptable solutions for HR 8799 d place the planet on an orbit inclined by more than $7^{\circ}\left(21^{\circ}\right)$ relative to HR $8799 \mathrm{~b}(\mathrm{c})$ 's orbit: few orbital solutions consistent with the astrometry also place them on coplanar orbits. Adopting a less restrictive definition for "acceptably fitting" orbits does not undo any of these trends, although there are more orbit combinations making the planets coplanar. Adopting the median parameter value or MLO instead of the more conservative $68 \%$ confidence interval likewise does not change these results.

These results provide valuable input for constraining the mass of the HR 8799 planetary system. Longer-term astrometric monitoring of HR 8799 (i.e., Konopacky et al. 2012) will better clarify the planets' orbital properties. Limits on the planets' dynamical masses will provide crucial input for planet cooling models and even more firmly establish HR 8799 as a benchmark system to understand the properties of young, self-luminous planets.

Finally, this work and other recent studies of HR 8799 (Soummer et al. 2011; Lafreniére et al. 2009; Fukagawa et al. 2009) clearly demonstrate the value of publicly archiving data on advanced telescopes. In our case, detecting at least two HR 8799 planets (HR 8799 bc) was rather straightforward and did not require advanced image processing techniques developed well after the data were taken. As data for Keck and many other 

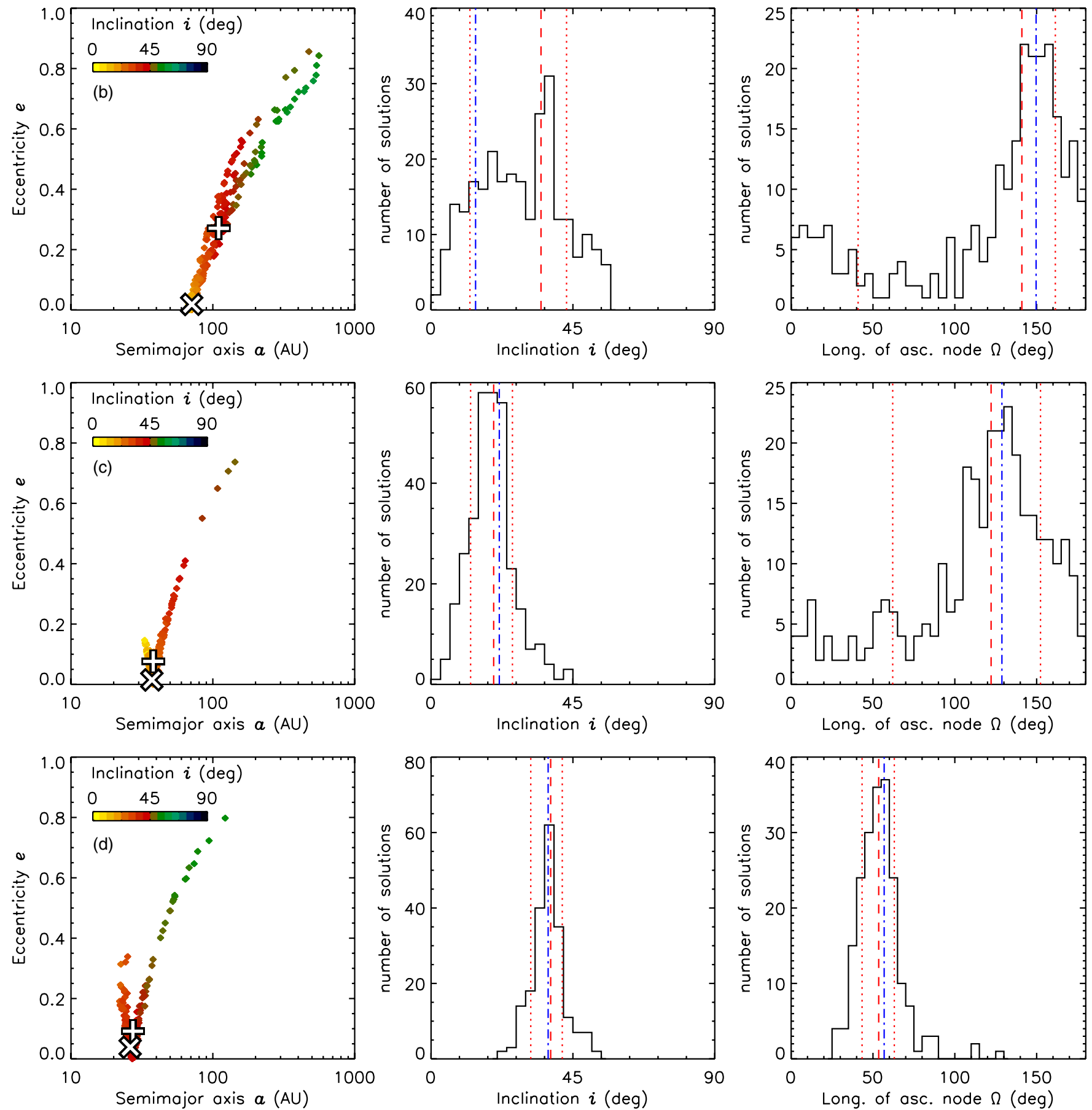

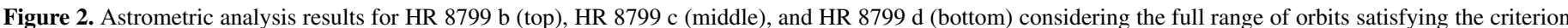

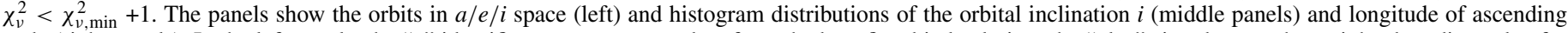

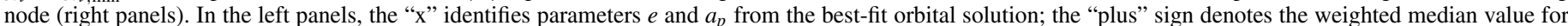

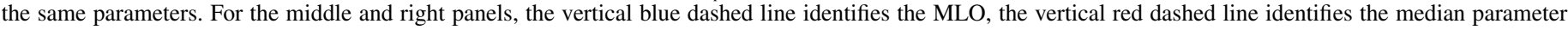
value, and the vertical red dotted lines bracket the $68 \%$ confidence interval.

(A color version of this figure is available in the online journal.)

8-10 m class telescopes are now archived, they provide an indispensable resource with which to confirm and characterize directly imaged planets like HR 8799's and other substellar companions.

We thank Christian Marois, Scott Kenyon, and the anonymous referee for helpful comments. This research has made use of the Keck Observatory Archive (KOA), which is operated by the W. M. Keck Observatory and the NASA Exoplanet Science Institute (NExScI), under contract with the National Aeronautics and Space Administration. We are extremely grateful to the NExScI/KOA staff for developing and maintaining the NIRC2 archive. T.C. is supported by a NASA Postdoctoral Fellowship; S.M. is supported by an Astronomy Center for Theory and Computation Prize Fellowship at the University of Maryland. 

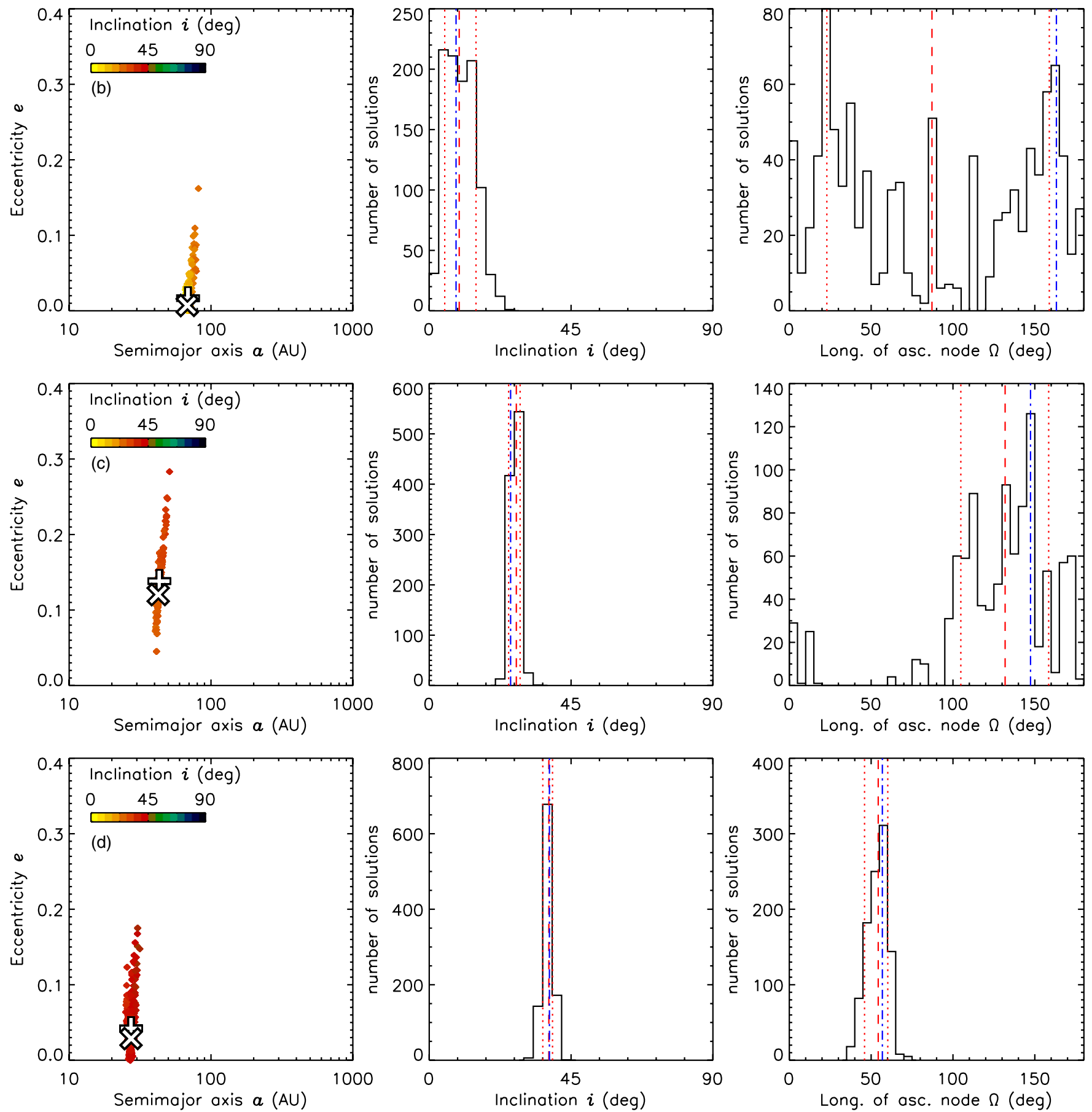

Figure 3. Same as Figure 2 except only for non-crossing orbits consistent with a 4:2:1 mean-motion resonance between HR 8799 b, c, and d, a configuration which promotes orbital stability (e.g., Fabrycky \& Murray-Clay 2010). Note the lack of high-eccentricity orbits and the narrower range in acceptable orbital parameters, especially for HR $8799 \mathrm{c}$ and $\mathrm{d}$.

(A color version of this figure is available in the online journal.)

\section{REFERENCES}

Currie, T., Bailey, V., Fabrycky, D., et al. 2010, ApJ, 721, L177

Currie, T., Burrows, A., Itoh, Y., et al. 2011a, ApJ, 729, 128

Currie, T., Rodigas, T., Debes, J., et al. 2012, ApJ, in press (arXiv:1206.4688)

Currie, T., Thalmann, C., Matsumura, S., et al. 2011b, ApJ, 736, 33L

Esposito, S., Mesa, D., Skemer, A., et al. 2012, A\&A, submitted (arXiv:1203.2735)

Fabrycky, D. C., Lissauer, J. J., Ragozzine, D., et al. 2012, ApJ, submitted (arXiv:1202.6328)

Fabrycky, D. C., \& Murray-Clay, R. A. 2010, ApJ, 710, 1408
Fukagawa, M., Itoh, Y., Tamura, M., et al. 2009, ApJ, 696, L1 Galicher, R., Marois, C., Macintosh, B., et al. 2011, ApJ, 739, L41

Konopacky, Q., et al. 2012, BAAS, 31, 2012

Lafreniére, D., Marois, C., Doyon, R., \& Barman, T. 2009, ApJ, 694, L148

Lafreniére, D., Marois, C., Doyon, R., Nadeau, D., \& Artigau, É. 2007, ApJ, 660,770

Madhusudhan, N., Burrows, A., \& Currie, T. 2011, ApJ, 737, 34

Marois, C., Lafrenière, D., Doyon, R., Macintosh, B., \& Nadeau, D. 2006, ApJ, 641, 556

Marois, C., Macintosh, B., Barman, T., et al. 2008, Science, 322, 1348

Marois, C., Macintosh, B., \& Véran, J.-P. 2010a, Proc. SPIE, 7736, 52 
Marois, C., Zuckerman, B., Konopacky, Q. M., Macintosh, B., \& Barman, T. 2010b, Nature, 468, 1080

Metchev, S., Marois, C., \& Zuckerman, B. 2009, ApJ, 705, L204

Rodigas, T. J., Hinz, P. M., Leisenring, J., et al. 2012, ApJ, 752, 57

Soummer, R., Brendan Hagan, J., Pueyo, L., et al. 2011, ApJ, 741, 55
Spiegel, D., \& Burrows, A. 2012, ApJ, 745, 174

Sudol, J., \& Haghighpour, N. 2012, ApJ, 755, 38

Thalmann, C., Carson, J., Janson, M., et al. 2009, ApJ, 707, L123

Yelda, S., Lu, J. R., Ghez, A. M., et al. 2010, ApJ, 725, 331

Zuckerman, B., Rhee, Joseph H., Song, I., \& Bessell, M. S. 2011, ApJ, 732, 61 